\title{
Rh Negative Blood Group
}

National Cancer Institute

\section{Source}

National Cancer Institute. Rh Negative Blood Group. NCI Thesaurus. Code C76252.

A blood group indicating the absence on erythrocytes of the Rh D form of the Rhesus antigen. 